\title{
Linearly decayed evanescent optical field in planar refractive index well
}

\author{
JIANHUA LIU, ${ }^{1 *}$ LI TAO ${ }^{2}$ \\ ${ }^{1}$ Department of Optical Science and Engineering, \\ Key Lab for Micro and Nanophotonic Structures (Ministry of Education), Fudan University, Shanghai 200433, China \\ Shanghai Ultra-Precision Optical Manufacturing Engineering Center, Fudan University, Shanghai 200438, China \\ ${ }^{2}$ Department of Electrical Engineering, Stanford University, Stanford, CA 94305, USA \\ *Corresponding author: jianhua Liu@fudan.edu.cn, 220 Handan Road, Shanghai, 200433, China
}

\begin{abstract}
Evanescent optical field with linearly decaying profile is theoretically analyzed at the critical angle of incidence in a planar structure of one dimensional refractive index well (RIW). The linearity of the evanescent field is due to the presence of the second refractive index barrier, which also shifts the position of total internal reflection (TIR) away from the critical angle. The decaying rate is determined by the refractive indices of the two barriers, as well as the width of the well. With this linearly decayed evanescent field (LDEF), various profiles across the well, for example uniform one, can be formed via appropriate combination of the LDEFs, which can promote new applications in fields of material analysis and sensing in the molecular scale.
\end{abstract}

Keywords: Inhomogeneous optical media; Total internal reflection; Tunneling; Sensors

\section{1. introduction}

Planar optical waveguide is usually composed of three uniform layers of different refractive indices (RIs), i.e., a guiding layer of higher RI in the center, and two surrounding layers of relatively lower RIs. This one dimensional structure can be called a planar refractive index ridge (RIR), which has been studied extensively in the late 1990's, and guided wave optics has been an important branch of optics [1]. On the other hand, the inverse structure, i.e., a lower refractive index layer sandwiched between two higher RI parts, which can be called a planar refractive index well (RIW), has not been equally studied as RIR, even though it has been used in reflection spectroscopies[2]. It is well known that when a beam of light is incident from a higher RI region to a lower RI one, i.e. the single boundary high-low RI structure, evanescent field can be generated in the lower RI region and decays exponentially from the boundary if the angle of incidence exceeds the critical angle. The critical angle is the incident angle at which total internal reflection (TIR) happens at the high-low RI boundary. If another high RI substrate is now put close to the first boundary in the lower RI region, which forms a one dimensional planar structure of RIW, the evanescent field inside the well would be different from that of the single-boundary high-low RI structure, especially at the critical angle of incidence.

Optical wave in a RIW structure has been discussed by Ulrich and Tian $[3,4]$ for the modelling of prism coupled planar optical waveguide. However their attention was mainly focused on the energy transferring from the prism into the waveguide, so that the optical field property was not emphasized. They categorized the coupling process into strong and weak regimes in terms of the coupling strength, which is a function of the thickness of the coupling gap. That makes their model imperfect for those cases when arbitrary gap thicknesses are concerned. Actually, optimal coupling efficiency does exist at certain gap thicknesses [5, 6]. Midwinter [7] presented another analysis method with no subjective restriction on the gap thickness. However, direct programing on Midwinter's model produces singularities if the gap thickness becomes overly large. Hansen [8] investigated the stratified structure, and obtained explicit expressions for the electric field of optical waves in especially the three layered ("three phase" [8]) structure. However, the field expression was for travelling wave, so that the evanescent situation was not included. Chilwell et al [6] analyzed the prism loaded planar optical waveguide structure by means of thinfilms field-transfer matrix theory. They cared more about the reflectance, and thus the optical field property inside the coupling medium was ignored.

Based on the Rigorous coupled wave analysis (RCWA) [9], we have analyzed the optical field in the RIW structure, and found that at the critical angle of incidence the evanescent field in the RIW decays linearly, instead of exponentially, across the gap. In addition, at the critical angle of incidence, the intensity reflectance at the coupling side is still lower than $100 \%$. With this linearly decayed evanescent field (LDEF), various types of evanescent field in the gap can be formed, which can find scientific and instrumental applications in fields of material analysis and optical sensing. 


\section{Modeling and Theory}

The one dimensional RIW is schematically depicted in Fig.1(a). The structure is composed of two high RI planar regions and a sandwiched uniform lower RI layer. The refractive indices for each region from the bottom to the top are labeled as $n_{s}, n_{g}$, and $n_{c}$, and the regions are denoted as the substrate, the gap, and the cladding, respectively. The refractive index profile across the RIW is illustrated in Fig.1(b). The gap thickness is d. A coordinate system is built as in the figure, with $\mathrm{X}$ axis along the normal of the gap interfaces, and $\mathrm{Z}$ axis along the wave "guiding" direction. The origin of the coordinate system is set at the lower boundary of the gap.

A beam of light is incident from within the substrate at an angle $\theta$. If $\theta$ is less than the critical angle ( $\theta$ c), the optical wave will transmit through the interfaces and get into the cladding region. If the incident angle is equal to or exceeds $\theta c$, the incident optical wave will be reflected completely at the boundary, and therefore evanescent field is generated and it is expected to decay exponentially into the gap. If the gap is wide enough, as categorized as the week coupling regime by Ulrich and Tien $[3,4]$, the incident optical waves cannot tunnel into the cladding region. However the situation for a RIW is to some extent different from the conventional description above, especially for the behavior of the evanescent field and for the onset of the TIR.
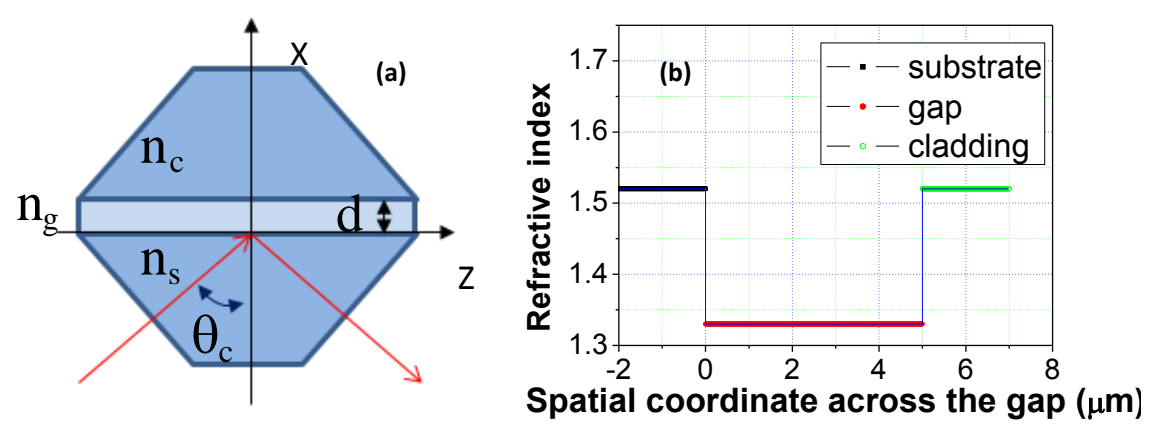

Fig1. (a) Schematic cross-sectional structure of a planar refractive index well (RIW). (b) Refractive index profile across the RIW structure.

We focus our attention on TE wave, i.e., the electric field of the incident optical beam is perpendicular to the XZ plane in Fig.1. According to RCWA[9], the optical fields in the three regions can be expressed respectively as:

$$
\begin{array}{lr}
E_{c}=\left(A_{c} e^{-\lambda_{c}(x-d)}\right) e^{-j \beta z} & x \geq d \\
E_{g}=\left(A_{g} e^{-\lambda_{g} x}+B_{g} e^{\lambda_{g}(x-d)}\right) e^{-j \beta z} & d \geq x \geq 0 \\
E_{s}=\left(A_{s} e^{-\lambda_{s} x}+B_{s} e^{\lambda_{s} x}\right) e^{-j \beta z} & x \leq 0
\end{array}
$$

where each quantity with subscript of 's', 'g', or ' $c$ ' is related to the substrate, gap, or the cladding region respectively. $E_{s}, E_{g}$, and $E_{c}$ are the optical field vectors in the corresponding regions, with $A_{s}$ and $B_{s}, A_{g}$ and $B_{g}$ representing the coefficients of the forward and backward eigen waves in the substrate and the gap regions. The cladding region is assumed infinitely thick, so that there is no backward wave, only forward coefficient $A_{c}$ exists. $\lambda_{s}, \lambda_{g}$, and $\lambda_{c}$ are the eigenvalues of optical waves in the corresponding regions. $\beta=k_{\text {on }}$ eff is the effective wave vector along the $\mathrm{Z}$ direction. $k_{0}=2 \pi / w$ is the wave vector with wavelength $w$ in vacuum. The effective refractive index is defined as $n_{e f f}=n_{s} \sin \theta$. The eigenvalue is defined as $\lambda_{i}=\sqrt{\beta^{2}-k_{0}^{2} n_{i}^{2}}$, where $\mathrm{i}=\mathrm{s}, \mathrm{g}$, $\mathrm{c}$.

According to the continuity condition [1] at the two boundaries of the gap, the relations of the coefficients of different regions can be obtained. The amplitude reflectivity and intensity reflectance in the substrate region can be defined as $r=B_{s} / A_{s}$, and $R=|r|^{2}$ respectively. The amplitude transmission coefficient and the intensity transmittance in the cladding region are defined as $t=A_{c} / A_{s}$, and $T=\frac{\lambda_{c}}{\lambda_{s}}|t|^{2}[10]$. Therefore the field coefficients in each region can be obtained in terms of the incident wave amplitude $A_{s}$. The optical field in each region of the RIW structure can then be obtained as a function of the incident angle $\theta$.

At the critical angle of incidence $\left(\theta_{c}\right), n_{e f f}=n_{s} \sin \theta_{c}=n_{g}$, which results in a zero eigen-value in the gap region, i.e. $\lambda_{g}=0$. Substituting directly this zero eigen-value into (1), we can acquire the optical field inside the gap $\left(E_{g}\right)$ as a constant that is independent of the spatial coordinate. This constant field in the gap is similar to that in the lower RI region in the case of total internal reflection at a single-boundary high-low RI structure. However for RIW, that constant field across the gap violates the boundary condition at the cladding side. 
To acquire the expression for the optical field in the gap at the critical angle of incidence $\left(\theta_{c}\right)$, the first order approximation is adopted, where the exponential function in the field expression $E_{g}$ in (1) is approximated by $e^{x} \approx 1+x$. With some steps of algebra, the optical field in the gap can be expressed as:

$$
E_{g}=\left(A_{s}+B_{s}\right)-\left(\frac{2 \lambda_{s} \lambda_{c}}{\lambda_{s}+d \lambda_{s} \lambda_{c}+\lambda_{c}} A_{s}\right) x
$$

which is a linearly decaying function on the spatial coordinate $\mathrm{x}$.

Applying the first order approximation at the critical angle, i.e., $\lambda_{g} \approx 0$, we derive the amplitude reflectivity as:

$$
r=\frac{\lambda_{s}+\lambda_{c} \lambda_{s} d-\lambda_{c}}{\lambda_{s}+\lambda_{c} \lambda_{s} d+\lambda_{c}}
$$

From equation (3), evanescent field represented by equation (2) can be definitely characterized with the help of $r=B_{s} / A_{s}$.

\section{Numerical example}

To elucidate the linearly decayed evanescent field (LDEF) in the gap of the RIW at the critical angle, an example of a planar symmetric RIW is shown below. We assume a structure of a layer of water sandwiched between two glass prisms, where refractive indices of the substrate and cladding regions are $n_{s}=n_{c}=1.52$, and that of the water layer is $n_{g}=1.33$. A He-Ne laser beam with wavelength of $632.8 \mathrm{~nm}$ is incident from the substrate side onto the boundary with the water gap. Fig.2(a) shows the amplitude ratios of the field coefficients $A_{g}$ and $B_{g}$ in the gap over that of the incident light $A_{s}$ in the substrate, as a function of the incident angle. The angle corresponding to the highest peak in Fig.2(a) is the critical angle, $\theta_{c}=61.0450^{\circ}$. Fig.2 (b) shows the intensity reflectance and transmittance of a glass-water-glass RIW as a function of the incident angle. It can be seen that around the critical angle, R and T get saturated gradually.
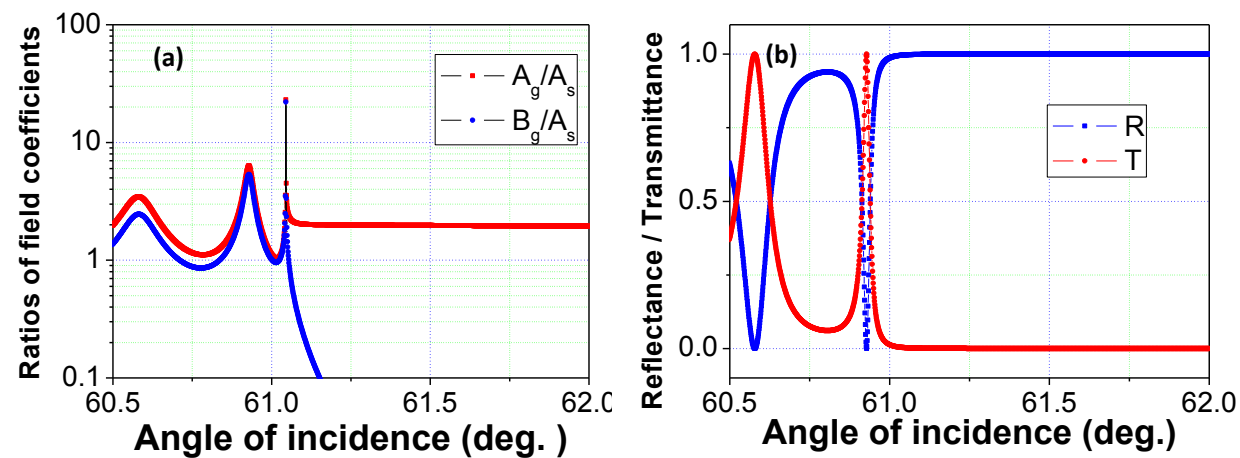

Fig.2 (a) Amplitude ratios of field coefficients $A_{g}$ and $B_{g}$ over $A_{s}$ for a glass-water-glass RIW structure. Red square points: for $A_{g} / A_{s}$, blue round points: for $B_{g} / A_{s}$ (b) Intensity reflectance and transmittance in the RIW as a function of the incident angle.

Fig.3 shows the field amplitude profile in the RIW structure at the critical angle of incidence. It can be seen that in the substrate region, the optical wave oscillates, which is due to the interference between the traveling waves to and from the boundary. The field in the water gap decays linearly to the cladding side, satisfying the continuity condition at both boundaries. The field in the cladding region is a single traveling wave because of the infinite thickness assumption.

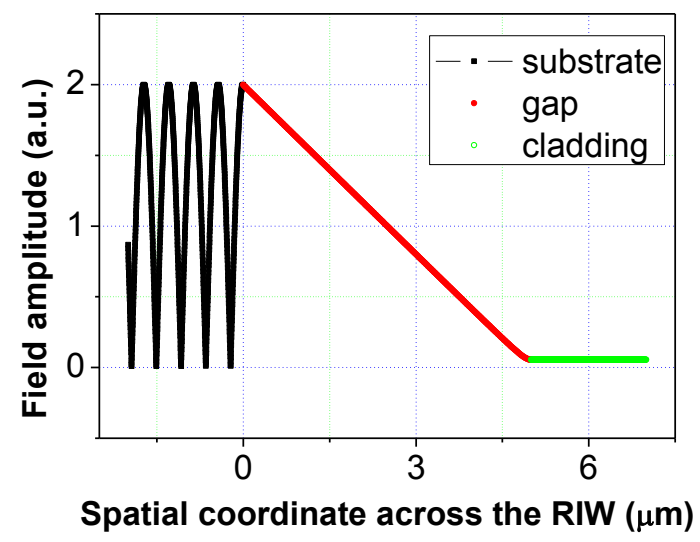

Fig.3 Field amplitude profile of the RIW structure at the critical angle of incidence $\theta c$. 
Since the incident angle is right at the critical angle $(\theta c)$, total internal reflection (TIR) should occur at the substrate-gap interface. However, from Fig.3, it can be seen that the amplitude in the cladding region is not completely zero, showing that TIR is still not set on even at the critical angle of incidence. Further assessment on the intensity reflectance (R) and transmittance (T) in Fig.2(b) shows that at the critical angle $\left(\theta c=61.0450^{\circ}\right), R \approx 0.997$, and $T \approx 0.003$ for this RIW structure. Checking the analytical expression from equation (3) for value of the reflection at the critical angle of incidence, it is clear that $r$ is not completely $100 \%$ (TIR) unless the gap thickness is approaching infinity.

It can be seen that as the value of the gap thickness (d) gets larger and larger, (2) and (3) both converge to constant values which are consistent with the single boundary high-low RI structure situation. Therefore, due to the presence of the cladding region at a finite gap distance, TIR is suppressed even at the critical angle that is defined by a single boundary high-low RI structure. As the incident angle increases to exceed the critical angle, the reflectance and transmittance get saturated to $100 \%$ and 0 , respectively, and the decaying rate of the evanescent field approaches completely exponential.

Analytically equation (2) gives the mathematical expression of the LDEF in the gap in the case that there is a tiny offset of the eigenvalue from the exact zero, i.e. $\lambda_{g} \approx 0$. Numerically this is practically true for an algorithm in a digital computer which has a finite precision of the data type. Even in the condition of an exact match of the effective refractive index to that of the gap, i.e., $n_{e f f}=n_{s} \sin \theta_{c}=n_{g}$, the calculated eigenvalue $\lambda_{g}$ is still not exactly zero. Therefore, no matter how carefully the RIW is theoretically designed in terms of the configuration on $\mathrm{n}_{\mathrm{s}}$ and $\mathrm{n}_{\mathrm{g}}$ at the critical angle of incidence, the numerical result of the evanescent field always decays linearly. Physically, this could be explained in terms of the deformation of the wave front in the gap by the squeeze of the cladding region at a finite gap distance. The effect of the presence of the cladding region is to bend the wave plane, even if to an infinitesimal extent, that a certain part (even though it might be tiny) of the wave vector in X direction is then produced, making $\lambda_{g}$ not exactly zero at the critical angle $\theta_{c}$. This phenomenon might find its similitude in the case of a beam of plane wave confronting an opaque plane with a hole of finite diameter. The rim of the hole bends the wave front of the incoming plane wave so that extra spatially spectral components are generated [11]. These phenomena are a type of the convolution effect originating from the oscillating nature of optical waves.

Although theoretically there is a high peak for coefficients $A_{g}$ and $B_{g}$ respectively for the field in the gap as demonstrated in Fig.2(a), TIR is still not triggered on at that peak, but gradually emerges in $\mathrm{R}$ or $\mathrm{T}$, without any critical indication, as the angle of incidence exceeds the critical angle, as demonstrated in Fig. 2(b). Therefore experimentally to locate the critical angle for obtaining the linear evanescent field in a RIW gap, we need to make sure that the reflectance on the substrate side just gets saturated and/or the transmittance in the cladding side just gets stably less than $1 \%$. The deviation of the slope of evanescent field from linearity in the gap can be estimated in terms of spatially averaged field amplitude. For a $5 \mu \mathrm{m}$ gap water-glass RIW, a $0.01^{\circ}$ increase from the critical angle would cause about $6 \%$ reduction in the averaged evanescent amplitude, which can be easily controlled by a mechanical rotational stage. The wider the gap, the more sensitive the linear field deviation will be. Therefore LDEF can be sustained without much difficulty in a RIW structure with the gap thickness less than $5 \mu \mathrm{m}$.

For a symmetric RIW structure, as the numerical example of this water sandwiched RIW, if it is coupled by two laser beams from both sides of the gap at the critical angle of incidence simultaneously, linearly decayed evanescent field from the corresponding side could be generated, as the two straight lines, LDEF left and LDEF right, as shown in Fig.4. Consequently a synthetic evanescent field in the gap could be completely uniform if the two coupling laser beams are in phase and of equal amplitude. This can be seen by the horizontal trace of 0 phase delay (synthesized 0) in Fig.4. If a $\pi$ phase delay exists between the two coupling beams, the evanescent field in the gap gets decreased in the central portion with a symmetric negative slope from the two sides, as the V shape trace (synthesized $\pi$ ) in Fig.4. With the control of different phase delay and amplitude ratio of the two coupling laser beams on the two sides of the RIW, various types of evanescent field profiles can be synthesized.

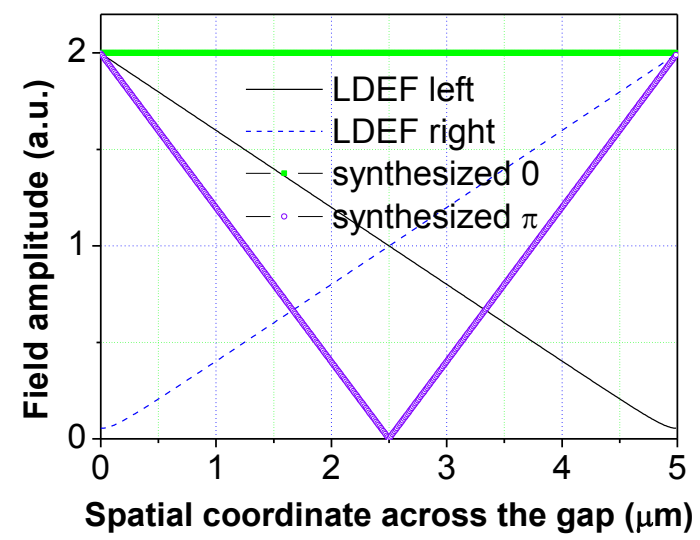

Fig.4 Field amplitude profile across the gap of a glass-water-glass RIW structure at the critical angle of incidence with laser beams coupled from both sides. Please refer to the text for trace details. 
Evanescent field excitation has been playing important roles in fluorescence and Raman scattering studies on molecular analytes at surface/interfaces $[12,13]$. Enhancement of evanescent field based on one dimensional photonic crystal (1DPC) supported Block surface wave (BSW) makes the evanescent field excitation a promising tool in micro and nano scale analysis on materials in molecular level, and in sensing applications [14-18]. However, due to the usually rapid exponentially decaying property, the optical field is inhomogeneous even in the range of penetration depth of the evanescent field [14], so that the signals to be detected are inhomogeneous, especially for molecules with 3d structures [12]. The LDEF analyzed above provides an option to build a homogeneous evanescent field that could be flexible for different analytes of micro to nanometer sizes, and could be a tool of sensing to those specimens contained in solutions or gases. As shown in Fig.4, evanescent field in RIW can be tailored, which will find suitable applications in molecular trace analysis and sensing.

\section{4. conclusion}

We have analytically and numerically demonstrated the linearly decayed evanescent field (LDEF) in a planar refractive index well at the critical angle of incidence. The linearity of the decay is due to the presence of the second refractive index barrier, which also shifts the position of the TIR away from the critical angle. The decay rate of the LDEF depends on the refractive indices of the two RI barriers and the thickness of the gap. Various profiles of evanescent fields in the RIW structure can be generated by means of simultaneous coupling of two laser beams symmetrically from both sides of the RIW structure. Uniform and other types of evanescent field can be constructed, which will find important applications in surface or interface based micro or nano scale Raman or Fluorescence measurements, as well as sensing.

This work is supported by the National Science Foundation of China under the contract of 61575047.

\section{References:}

1. A. Yariv and P. Yeh, Optical waves in crystals, John Wiley \& Sons, New York, 1984

2. A. Hjortsberg, W. P. Chen, and E. Burstein, "Resonant internal-reflection prism spectroscopy using surface, guided, and Fabry-Perot EM waves", Applied Optics, 17(3/1), (1978), 430-4,

3. R.Ulrich, "Theory of the Prism-Filn Coupler by Plane-Wave Analysis", J. Opt. Soc. Am, 60(10), 1337, 50, 1970

4. $\quad$ P. K. Tien, and R.Ulrich, "Theory of Prism-Film Coupler and Thin-Film Light Guides", J. Opt. Soc. Am, 60(10), (1970), 1325-37

5. $\quad$ R. ULRICH, "Optimum Excitation of Optical Surface Waves", J. Opt. Soc. Am. 61(11), (1971), 1467-77

6. John Chilwell, and Ian Hodgkinson, "Thin-films field-transfer matrix theory of planar multilayer waveguides and reflection from prism-loaded waveguides", J. Opt. Soc. Am. A, 1(7), (1984), 742-53

7. J. E. MIDWINTER, IEEE, “Evanescent Field Coupling into a Thin-Film Waveguide”, J. Quan. Elect., QE-6(10), (1970), 583-90

8. W. N. Hansen, "Electric Fields Produced by the Propagation of Plane Coherent Electromagnetic Radiation in a Stratified Medium", J. Opt. Soc. Am. 58(3), (1968), 380-90

9. M. G. Moharam, Drew A. Pommet, and Eric B. Grann, T. K. Gaylord, "Stable implementation of the rigorous coupled-wave analysis for surfacerelief gratings: enhanced transmittance matrix approach", J. Opt. Soc. Am. A, 12(5), (1995), 1077-86

10. M. Born, and E. Wolf, principles of optics, Cambridge, 7th ed. , p.43, 2007

11. Joseph W. Goodman, Introduction to Fourier Optics, McGraw-Hill, Inc., 1968

12. S. Pirotta, X. G. Xu, A. Delfan, S. Mysore, S. Maiti, G. Dacarro, M. Patrini, M. Galli, G. Guizzetti, D. Bajoni, J. E. Sipe, G. C. Walker, and M. Liscidini, "Surface-Enhanced Raman Scattering in Purely Dielectric Structures via Bloch Surface Waves",

J. Phys. Chem. C 2013, 117, 6821-6825,

13. De-Bo Hu, Chen Chen, and Zhi-Mei Qi, "Resonant Mirror Enhanced Raman Spectroscopy”, J. Phys. Chem. C 2014, 118, 13099-13106

14. C. Ndiaye, M. Zerrad, A. L. Lereu, R. Roche, Ph. Dumas, F. Lemarchand, and C. Amra, "Giant optical field enhancement in multi-dielectric stacks by photon scanning tunneling microscopy", Appl. Phys. Lett. 103, (2013), 131102

15. W. M. Robertsona and M. S. May, "Surface electromagnetic wave excitation on one-dimensional photonic band-gap arrays", Appl. Phys. Lett. 74(13), (1999),1800-2

16. I.V.Soboleva, E. Descrovi, C. Summonte, A. A. Fedyanin, and F. Giorgis, "Fluorescence emission enhanced by surface electromagnetic waves on one-dimensional photonic crystals", Appl. Phys. Lett. 94, (2009), 231122

17. Francesco Michelotti and Emiliano Descrovi, "Temperature stability of Bloch surface wave biosensors", Appl. Phys. Lett. 99, (2011), 231107

18. L. Fornasari, F. Floris, M. Patrini, G. Canazza, G. Guizzetti, D. Comoretto, and F. Marabelli, "Fluorescence excitation enhancement by Bloch surface wave in all-polymer one-dimensional photonic structur",

Appl. Phys. Lett. 105, (2014), 053303 